\title{
Stability of fractional order parabolic partial differential equations using discretised backstepping method
}

\author{
Ibtisam Kamil Hanan a, b, Muhammad Zaini Ahmad ${ }^{\text {a, }}$, Fadhel Subhi Fadhel ${ }^{\text {b }}$ \\ a Institute of Engineering Mathematics, Universiti Malaysia Perlis, Pauh Putra Main Campus, 02600 Arau, Perlis, Malaysia \\ b Department of Mathematics and Computer Applications, College of Science, Al-Nahrain University, P. O. Box 47077, Baghdad, Iraq \\ * Corresponding author: mzaini@unimap.edu.my
}

\section{Article history}

Received 2 August 2017

Accepted 5 November 2017

\begin{abstract}
This paper focuses on the application of backstepping control scheme for fractional order partial differential equations (FPDEs) of order $\beta$ with $1<\beta \leq 2$. Therefore to obtain highly accurate approximations for this derivative is of great importance. Here the discretised approach for the space variable is used to transform the FPDEs into a system of differential equations. These approximations arise mainly from the Caputo definition and the Grünwald-Letnikov definition. A Lyapunov function is defined at each stage and the negativity of an overall Lyapunov function is ensured by proper selection of the control law. Illustrative example is given to demonstrate the effectiveness of the proposed control scheme.

Keywords: Backstepping method, Lyapunov function, Fractional derivative
\end{abstract}

\section{INTRODUCTION}

The concept of fractional derivative dates back to the 17 th century [1-3]. The fractional-order operators now serve as excellent tools for the mathematical modelling of real world problems [4]. The fractional order differential equations as ordinary and partial cases has been involved in a wide range of real life problems, such as the applications in fluid dynamics, biology, viscoelasticity, physics and engineering. Therefore, the solution of these fractional order differential equations of physical interest has gain a huge amount of attention. [5-9].

The concept of fractional calculus has interacted with the control community deeply during the last few decades. That interaction is based on two major factors, the first is that we cannot describe many of engineering plants and processes without involving the fractional order calculus $[10,11]$. In addition, the fractional order controller is proved to be given a more freedom in the design. The concept of fractional order systems has been perfectly covered and there are a very large range of applications and their outcomes have been obtained [12,13]. For more details one can find some excellent references in [11,13,14].

The backstepping control is one category of control approaches that has gain a considerable attention in the case of controlling parametric nonlinear strict feedback systems. It is based control approach which is a recursive design technique that breaks down the control design for the full system into a sequence of lower-order subsystems $[15,16]$. Backstepping is unlike any of the methods previously developed in literatures for controlling ordinary differential equations (ODEs) and partial differential equations (PDEs). It differs from optimal control methods in that it sacrifices optimality (though it can achieve a form of "inverse optimality") for the sake of avoiding the operator of Riccati equations, which are very hard to solve for infinite or high dimensional systems, such as PDEs. Backstepping is also different from pole placement methods, because even though its objective is the stabilisation of the system, which is also the same objective of the pole placement methods. In addition, backstepping does not pursue precise assignment of even a finite subset of the PDE's eigenvalues [17]. The scheme of the backstepping control is basically a recursive controller that uses virtual controls (which are a set of intermediate variables), constructed the Lyapunov function from the total closed-loop control system. The backstepping technique in the integer order has been extensively studied because of the huge advantages the technique gives, such as global stability, good tracking and transient performance. However, it is been very few research in the literature that is succeeded to apply the backstepping method on the case of the fractional order system. For instance, for the first time, Efe [18] has tried to extend the backstepping technique to fractional order systems. Next, Sahab [19] has implemented a generalization backstepping method in order to approximate a solution of the fractional order error differential equation with respect to two new fractional order hyperchaotic system. In [20], the author has used the backstepping method to describe and design a controller for a fractional order chaotic system control issue.

Recently we proposed the backstepping method for stabilising time fractional order PDE. The semi-discretised fractional-order backstepping approach introduced to find the boundary controller function which stabilises the time fractional order PDE by transformation it into an equivalent stable closed loop [21]. In this paper a discretised backstepping approach will be introduced to find the boundary controller function which stabilises the nonlinear PDE with space fractional derivative by transformation it into an equivalent stable closed loop. We describe and compare two approximations of 
fractional derivative namely Caputo definition and GrünwaldLetnikov definition.

The rest of this paper is organised as follows: Some definitions for fractional order calculus are listed in Section two. In Section three we illustrate the backstepping approach to stabilise FPDE based on Lyapunov function. Finally, Section four provides an example and the result is illustrated and compared using two types of approximation for fractional derivative. The conclusions are devoted in the last section.

\section{FRACTIONAL DERIVATIVES}

In this section, we introduce definitions of fractional derivative which are used further in this paper.

Definition 1 [ 22]: The Caputo space-fractional derivative operator of order $\beta>0$ is defined as

$D_{x}^{\beta} s(x, t)=\frac{\partial^{\beta} s(x, t)}{\partial x^{\beta}}\left\{\begin{array}{lr}\frac{1}{\Gamma(m-\beta)} \int_{0}^{x}(x-\theta)^{m-\beta-1} \frac{\partial^{m} s(\theta, t)}{\partial \theta^{m}} d \theta & \text { for } m-1<\beta<m \\ \frac{\partial^{m} s(x, t)}{\partial x^{m}} & \text { for } \beta=m \in \mathrm{N}\end{array}\right.$

Definition 2 [23]: The Grünwald definition for the fractional derivative is

$D_{x}^{\beta} s(x)=\lim _{\Delta x \rightarrow 0} \frac{1}{\Delta x^{\beta}} \sum_{k=0}^{\left[\frac{x-a}{\Delta x}\right]}(-1)^{k}\left(\begin{array}{l}\beta \\ k\end{array}\right) s(x-k \Delta x)$.

\section{BACKSTEPPING METHOD FOR FPDE}

Consider the following nonlinear fractional order parabolic partial differential equation

$$
\frac{\partial s(x, t)}{\partial t}=\frac{\partial^{\beta} s(x, t)}{\partial x^{\beta}}+f(s(x, t)), 0<x<1, t \geq 0,
$$

(3)

where the fractional order $\beta$ belong to $(1,2], s \in L^{2}(\Omega)$, $\Omega=(0,1) \times[0, \mathrm{~T}], \mathrm{T}>0$ and $f$ is a nonlinear functions of $s$, such that $f \in C^{\infty}(R)$. With initial condition

$s(x, 0)=g(x), 0<x<1$.

The boundary condition at $x=0$ is homogenous Dirichlet $s(0, t)=0, \quad t \geq 0$,

and the boundary condition at other end

$s(1, t)=U(t)$,

where $U(t): C[0,1] \rightarrow R$ is the unknown nonlinear feedback control function to be design to achieve stabilisation.

We propose the bakstepping technique to stabilise the system (3)(6). A recursive design with Caputo derivative definition for discretising $\frac{\partial^{\beta} s(x, t)}{\partial x^{\beta}}$ is provided in case1 while the shifted GrünwaldLetnikov approximation is provided in case 2 . In these two cases the backstepping design technique is applied to obtain the boundary control function $U(t)$.

\section{Case1: Backstepping technique with Caputo approximation for FPDE}

The design procedure is divided into three stages as follows.

In the first stage, the nonlinear fractional parabolic partial differential equation in Eq. (3) will be semi-discretised into an equivalent nonlinear system of differential equations as follows:

Fix $N \in \mathbb{N}$ and $h=\frac{1}{n+1}$ as the step size of discretisation of system (3)-(6) over the interval of the space variable $x \in(0,1)$. Also, let $s_{i}(t)=s(i h, t)$ for all $i=0,1, \ldots, n+1$ where it is assumed that $s_{0}(t)$ is the first boundary condition and $s_{n+1}(t)$ is the control function to be evaluated, such that the original system is asymptotically stable. The semi-discretisng version of system (3)-(6) using the Caputo derivative definition is

$s_{0}(t)=0$,

$\frac{d s_{i}}{d t}=\frac{h^{-\beta}}{\Gamma(3-\beta)} \sum_{j=0}^{i-1} \mu_{i, j}\left(s_{j+2}-2 s_{j+1}+s_{j}\right)+f\left(s_{i}\right), \quad i=1,2, \ldots, n$,

$s_{n+1}=U(t)$,

where $\mu_{i, j}=(i-j)^{2-\beta}-(i-j-1)^{2-\beta}$. We can write the nonlinear semi-discretised system of differential equations as;

$$
\begin{aligned}
\frac{d s_{1}}{d t}= & \frac{h^{-\beta} \mu_{1,0}}{\Gamma(3-\beta)} s_{2}-\frac{2 h^{-\beta} \mu_{1,0}}{\Gamma(3-\beta)} s_{1}+f\left(s_{1}\right), \\
\frac{d s_{2}}{d t}= & \frac{h^{-\beta} \mu_{2,1}}{\Gamma(3-\beta)} s_{3}+\frac{h^{-\beta}\left(\mu_{2,0}-2 \mu_{2,1}\right)}{\Gamma(3-\beta)} s_{2}+\frac{h^{-\beta}\left(-2 \mu_{2,0}+\mu_{2,1}\right)}{\Gamma(3-\beta)} s_{1} \\
& +f\left(s_{2}\right),
\end{aligned}
$$

$$
\begin{aligned}
\frac{d s_{n}}{d t}= & \frac{h^{-\beta} \mu_{n, n-1}}{\Gamma(3-\beta)} U+\frac{h^{-\beta} \mu_{1,0}}{\Gamma(3-\beta)}\left(s_{2}-2 s_{1}\right)+\frac{h^{-\beta} \mu_{n, 1}}{\Gamma(3-\beta)}\left(s_{3}-2 s_{2}+s_{1}\right) \\
& +\cdots+\frac{h^{-\beta} \mu_{n, n-1}}{\Gamma(3-\beta)}\left(-2 s_{n}+s_{n-1}\right)+f\left(s_{n}\right) .
\end{aligned}
$$

In the second stage we will design the need controller according the idea of backstepping. The backstepping design procedure requires $n$ steps, and the virtual control $\alpha_{i}$ and the controller $U$ will be constructed. The design procedure is elaborated in the following theorem.

Theorem 1. Consider the system (10) with order $1<\beta \leq 2$ and $\frac{h^{-\beta}}{\Gamma(3-\beta)} \neq 0$ for $s \in R^{n}$ and $U: C[0,1] \rightarrow R$ is the nonlinear control functional. If the control Lyapunov function is taken by;

$v\left(w_{1}, w_{2}, \ldots, w_{n}\right)=\frac{h^{\beta}}{2} \sum_{i=1}^{n} w_{i}^{2}$,

where $w_{1}=s_{1}, w_{i}=s_{i}-\alpha_{i-1}, i=1,2, \ldots, n$ and $h=\frac{1}{n+1}>0$ is the step size of discretisation of system (3)-(6), that is there exist a nonlinear feedback control $U$ which renders the closed loop system asymptotically stable. The nonlinear feedback control functional can be designed as

$$
\begin{aligned}
U= & \left(2-k_{n} \Gamma(3-\beta)\right) s_{n}-2 s_{n-1}-\sum_{j=0}^{n-2} \mu_{n, j}\left(s_{j+2}-2 s_{j+1}+s_{j}\right)- \\
& \left.h^{\beta} f\left(s_{n}\right)+\alpha_{n-2}+k_{n} \Gamma(3-\beta) \alpha_{n-1}+h^{\beta} \Gamma(3-\beta) \sum_{j=1}^{n-1} \frac{\partial \alpha_{n-1}}{\partial s_{j}} \frac{\partial s_{j}}{\partial t}\right) . \\
\alpha_{i}= & \Gamma(3-\beta)\left(-k_{i} w_{i}-\frac{1}{\Gamma(3-\beta)} w_{i-1}-\frac{1}{\Gamma(3-\beta)} \sum_{j=0}^{i-2} \mu_{i, j}\left(s_{j+2}-2 s_{j+1}+s_{j}\right)\right. \\
& \left.-\frac{\mu_{i, i-1}}{\Gamma(3-\beta)}\left(-2 s_{i}+s_{i-1}\right)-h^{\beta} f\left(s_{i}\right)+h^{\beta} \sum_{j=1}^{i-1} \frac{\partial \alpha_{i-1}}{\partial s_{j}} \frac{\partial s_{j}}{\partial t}\right), i=3, \ldots, n-1
\end{aligned}
$$

where $k_{1}, \ldots, k_{n}>0$ are constants, $\mu_{i, j}=(i-j)^{2-\beta}-(i-j-1)^{2-\beta}$. proof. By the use of recursion, we havet he following steps; Step1: We start with the first equation in system (10). Design a suitable stabilising function $\alpha_{1}$ to stabilise $w_{1}(t)$. Select the Lyapunov function as $v_{1}=\frac{h^{\beta}}{2} w_{1}^{2}$.

Then the derivative of $v_{1}$ is given by 


$$
\begin{aligned}
\dot{v}_{1}= & -k_{1} w_{1}^{2}+\frac{1}{\Gamma(3-\beta)} w_{1} w_{2}+w_{1}\left(\frac{1}{\Gamma(3-\beta)} \alpha_{1}+k_{1} w_{1}-\right. \\
& \left.\frac{2}{\Gamma(3-\beta)} w_{1}+h^{\beta} f\left(s_{1}\right)\right) .
\end{aligned}
$$

The virtual control law $\alpha_{1}$ is designed as

$\alpha_{1}=\left(2-\Gamma(3-\beta) k_{1}\right) s_{1}-h^{\beta} \Gamma(3-\beta) f\left(s_{1}\right)$,

where $k_{1}>0$ is a design parameter. Then the resulting derivative is

$\dot{v}_{1}=-k_{1} w_{1}^{2}+\frac{1}{\Gamma(3-\beta)} w_{1} w_{2}, k_{1}>0$.

Based on LaSalle invariance principle, if $w_{2}=0$, then $w_{1}$ is guaranteed to converge to zero asymptotically.

Step2: Study the second equation of system (10) by considering $\alpha_{2}$ as a virtual control variable. The control objective is to make $w_{2} \rightarrow 0$ as $t \rightarrow \infty$. Define a control Lyapunov function as

$v_{2}=v_{1}+\frac{h^{\beta}}{2} w_{2}^{2}$

and its time derivative is given by;

$$
\begin{aligned}
\dot{v}_{2}= & -k_{1} w_{1}^{2}-k_{2} w_{2}^{2}+\frac{1}{\Gamma(3-\beta)} w_{2} w_{3}+w_{2}\left(\frac{1}{\Gamma(3-\beta)} \alpha_{2}+\frac{1}{\Gamma(3-\beta)} w_{1}\right. \\
& +k_{2} w_{2}+\frac{\left(\mu_{2,0}-2 \mu_{2,1}\right)}{\Gamma(3-\beta)} s_{2}+\frac{\left(-2 \mu_{2,0}+\mu_{2,1}\right)}{\Gamma(3-\beta)} s_{1}+h^{\beta} f\left(s_{2}\right)- \\
& \left.h^{\beta} \frac{\partial \alpha_{1}}{\partial s_{1}} \frac{\partial s_{1}}{\partial t}\right)
\end{aligned}
$$

By selecting

$$
\begin{aligned}
\alpha_{2}= & \Gamma(3-\beta)\left(-k_{2} w_{2}-\frac{1}{\Gamma(3-\beta)} w_{1}-\frac{\left(\mu_{2,0}-2 \mu_{2,1}\right)}{\Gamma(3-\beta)} s_{2}-\right. \\
& \left.\frac{\left(-2 \mu_{2,0}+\mu_{2,1}\right)}{\Gamma(3-\beta)} s_{1}-h^{\beta} f\left(s_{2}\right)+h^{\beta} \frac{\partial \alpha_{1}}{\partial s_{1}} \frac{\partial s_{1}}{\partial t}\right)
\end{aligned}
$$

where $k_{2}>0$, is the design parameter, then the resulting derivative of $v_{2}$ is

$\dot{v}_{2}=-\sum_{i=1}^{2} k_{i} w_{i}^{2}+\frac{1}{\Gamma(3-\beta)} w_{2} w_{3}$

if $w_{3}=0$, then $w_{2}$ is guaranteed to converge to zero asymptotically.

Step $i(i=3, \ldots, n-1)$ Study the ith equation of of system (10) with the virtual control variable $\alpha_{i}$. The control Lyapunov function is chosen as

$$
v_{i}=v_{i-1}+\frac{h^{\beta}}{2} w_{i}^{2},
$$

Its time derivative is given by

$$
\begin{aligned}
\dot{v}_{i}= & -\sum_{j=1}^{i} k_{j} w_{j}^{2}+\frac{1}{\Gamma(3-\beta)} w_{i} w_{i+1}+w_{i}\left(\frac{1}{\Gamma(3-\beta)} \alpha_{i}+k_{i} w_{i}+\frac{1}{\Gamma(3-\beta)} w_{i-1}\right. \\
& +\frac{1}{\Gamma(3-\beta)} \sum_{j=0}^{i-2} \mu_{i, j}\left(s_{j+2}-2 s_{j+1}+s_{j}\right)+\frac{\mu_{i, i-1}}{\Gamma(3-\beta)}\left(-2 s_{i}+s_{i-1}\right) \\
& \left.+h^{\beta} f\left(s_{i}\right)-h^{\beta} \sum_{j=1}^{i-1} \frac{\partial \alpha_{i-1}}{\partial s_{j}} \frac{\partial s_{j}}{\partial t}\right) .
\end{aligned}
$$

If we choose $\alpha_{i}$ as given in Eq. (13), $w_{i+1}$ is governed to zero. Then the resulting derivative of $v_{i}$ is

$$
\dot{v}_{i}=-\sum_{j=1}^{i} k_{j} w_{j}^{2}+\frac{1}{\Gamma(3-\beta)} w_{i} w_{i+1}
$$

At this point one can conclude that $w_{i}$ converge to zero asymptotically.
Step $n$ : In the last step $n$, the actual control $U$ appears and is at our disposal. The aim is that design a suitable control law to make $w_{n} \rightarrow 0$ as $t \rightarrow \infty$, select the Lyapunov function as

$v_{n}=v_{n-1}+\frac{h^{\beta}}{2} w_{n}^{2}$,

Then we can obtain the time derivative as

$$
\begin{aligned}
\dot{v}_{n}= & -\sum_{j=1}^{n} k_{j} w_{j}^{2}+w_{n}\left(\frac{1}{\Gamma(3-\beta)} U+k_{n} w_{n}+\frac{1}{\Gamma(3-\beta)} w_{n-1}\right. \\
& +\frac{1}{\Gamma(3-\beta)} \sum_{j=0}^{n-2} \mu_{n, j}\left(s_{j+2}-2 s_{j+1}+s_{j}\right) \frac{\mu_{n, n-1}}{\Gamma(3-\beta)}\left(-2 s_{n}+s_{n-1}\right) \\
& \left.+h^{\beta} f\left(s_{n}\right)-h^{\beta} \sum_{j=1}^{n-1} \frac{\partial \alpha_{n-1}}{\partial s_{j}} \frac{\partial s_{j}}{\partial t}\right) .
\end{aligned}
$$

One control can chosen by Eq. (12). So we have

$\dot{v}_{n}=-\sum_{i=1}^{n} k_{i} w_{i}^{2}$,

Consequently, $w_{n}$ is guaranteed to converge to zero asymptotically.

In the third stage substitute $U(t)$ evaluated by Eq. (12) back into system (10), for $i=n$, a system of $n$ nonlinear differential equations is obtained. The resulting system may be solved by using any method for solving a nonlinear system of differential equations.

\section{Case2: Backstepping technique with shifted Grünwald- Letnikov approximation for FPDE}

The design procedure that may be followed in general as we make in case1. Using the shifted Grünwald-Letnikov formula for discretising $\frac{\partial^{\beta} s(x, t)}{\partial x^{\beta}}$, which yields to;

$s_{0}(t)=0$,

$\frac{d s_{i}}{d t}=\frac{1}{h^{\beta}} \sum_{j=0}^{i+1} \mu_{j}^{(\beta)} s_{i-j+1}+f\left(s_{i}\right), \quad i=1, \ldots, n$,

$s_{n+1}=U(t)$,

where $\mu_{j}^{(\beta)}=(-1)^{j}\left(\begin{array}{l}\beta \\ j\end{array}\right)=(-1)^{j} \frac{\beta(\beta-1) \cdots(\beta-j+1)}{j !}$.

Similar to the first approximation. The nonlinear semi-discretised system of differential equations is;

$\frac{d s_{1}}{d t}=\frac{1}{h^{\beta}} s_{2}+\frac{\mu_{1}^{(\beta)}}{h^{\beta}} s_{1}+f\left(s_{1}\right)$,

$\frac{d s_{2}}{d t}=\frac{1}{h^{\beta}} s_{3}+\frac{\mu_{1}^{(\beta)}}{h^{\beta}} s_{2}+\frac{\mu_{2}^{(\beta)}}{h^{\beta}} s_{1}+f\left(s_{2}\right)$,

$\frac{d s_{n}}{d t}=\frac{1}{h^{\beta}} U+\frac{\mu_{1}^{(\beta)}}{h^{\beta}} s_{n}+\frac{\mu_{2}^{(\beta)}}{h^{\beta}} s_{n-1}+\cdots+\frac{\mu_{n}^{(\beta)}}{h^{\beta}} s_{1}+f\left(s_{n}\right)$.

we will design the need controller according to the idea of backstepping in the following theorem.

and

Theorem 2: Consider the system (29) with order $1<\beta \leq 2$ $\frac{1}{h^{\beta}} \neq 0$ for $s \in R^{n}$ and $U: C[0,1] \rightarrow R$ is the nonlinear control functional. If the control Lyapunov function is taken by Eq. (11), then there exist a nonlinear feedback control $U$ which renders the closed loop system asymptotically stable. The nonlinear control functional can be chosen by;

$$
\begin{aligned}
U= & \left(-k_{n} s_{n}-s_{n-1}-\sum_{j=1}^{n} \mu_{j}^{(\beta)} s_{n-j+1}-h^{\beta} f\left(s_{n}\right)+k_{n} \alpha_{n-1}+\alpha_{n-2}+\right. \\
& \left.h^{\beta} \sum_{j=1}^{n-1} \frac{\partial \alpha_{n-1}}{\partial s_{j}} \frac{\partial s_{j}}{\partial t}\right) .
\end{aligned}
$$


$\alpha_{i}=-k_{i} w_{i}-w_{i-1}-\sum_{j=1}^{i} \mu_{j}^{(\beta)} s_{i-j+1}-h^{\beta} f\left(s_{i}\right)+h^{\beta} \sum_{j=1}^{i-1} \frac{\partial \alpha_{i-1}}{\partial s_{j}} \frac{\partial s_{j}}{\partial t}, i=3, \ldots, n-1$,

where $k_{1}, \ldots, k_{n}>0$ are constants, $\mu_{j}^{(\beta)}=(-1)^{j} \frac{\beta(\beta-1) \cdots(\beta-j+1)}{j !}$. proof. The design procedure is given in the following steps.

Step1: The first Lyapunov functional is $v_{1}=\frac{h^{\beta}}{2} w_{1}^{2}$, and its derivative is $\dot{v}_{1}=-k_{1} w_{1}^{2}+w_{1} w_{2}+w_{1}\left(\alpha_{1}+k_{1} w_{1}+\mu^{(\beta)} s_{1}+h^{\beta} f\left(S_{1}\right)\right)$.

By selecting

$\alpha_{1}=-k_{1} w_{1}-\mu_{1}^{(\beta)} s_{1}-h^{\beta} f\left(s_{1}\right)$.

$w_{2}$ is to be governed to zero. Then, $\dot{v}_{1}=-k_{1} w_{1}^{2}+w_{1} w_{2}, k_{1}>0$

Step 2: consider the Lyapunov function $v_{2}=v_{1}+\frac{h^{\beta}}{2} w_{2}^{2}$, and its derivative given by

$$
\begin{aligned}
\dot{v}_{2}= & -k_{1} w_{1}^{2}-k_{2} w_{2}^{2}+w_{2} w_{3}+w_{2}\left(\alpha_{2}+w_{1}+k_{2} w_{2}+\mu_{1}^{(\beta)} \varepsilon_{2}+\mu_{2}^{(\beta)} s+\right. \\
& \left.h^{\beta} f\left(s_{2}\right)-h^{\beta} \frac{\partial \alpha_{1}}{\partial s_{1}} \frac{\partial s_{1}}{\partial t}\right),
\end{aligned}
$$

If the virtual control law $\alpha_{2}$ is designed as;

$\alpha_{2}=\left(-w_{1}-k_{2} w_{2}-\mu_{1}^{(\beta)} s_{2}-\mu_{2}^{(\beta)} s_{1}-h^{\beta} f\left(s_{2}\right)+h^{\beta} \frac{\partial \alpha_{1}}{\partial s_{1}} \frac{\partial s_{1}}{\partial t}\right)$,

$w_{3}$ is to be governed to zero. Then, $\dot{v}_{2}=-\sum_{i=1}^{2} k_{i} w_{i}^{2}$, where $k_{i}>0, i=1,2$ are the design parameters.

Step $i(i=3, \ldots, n-1)$ Study the ith equation of Eq.(29) with the virtual control variable $\alpha_{i}$. The control Lyapunov function is chosen as $v_{i}=v_{i-1}+\frac{h^{\beta}}{2} w_{i}^{2}$, Its time derivative is given by;

$$
\begin{aligned}
\dot{v}_{i}= & -\sum_{j=1}^{i} k_{j} w_{j}^{2}+w_{i} w_{i+1}+w_{i}\left(\alpha_{i}+k_{i} w_{i}+w_{i-1}+\sum_{j=1}^{i} \mu_{j}^{(\beta)} s_{i-j+1}+\right. \\
& \left.h^{\beta} f\left(s_{i}\right)-h^{\beta} \sum_{j=1}^{i-1} \frac{\partial \alpha_{i-1}}{\partial s_{j}} \frac{\partial s_{j}}{\partial t}\right) .
\end{aligned}
$$

If we choose $\alpha_{i}$ as given in Eq. (31), $w_{i+1}$ is governed to zero. Then we have

$$
\dot{v}_{i}=-\sum_{j=1}^{i} k_{j} w_{j}^{2}+w_{i} w_{i+1}
$$

Step $n$ : In the last step $n$, the actual control $U$ appears and is at our disposal. The aim is that design a suitable control law to make $w_{n} \rightarrow 0$ as $t \rightarrow \infty$, select the Lyapunov function as $v_{n}=v_{n-1}+\frac{h^{\beta}}{2} w_{n}^{2}$, then we can obtain the time derivative as;

$$
\begin{aligned}
\dot{v}_{n}= & -\sum_{j=1}^{n} k_{j} w_{j}^{2}+w_{n}\left(U+k_{n} w_{n}+w_{n-1}+\sum_{j=1}^{n} \mu_{j}^{(\beta)} s_{n-j+1}+h^{\beta} f\left(s_{n}\right)\right. \\
& \left.-h^{\beta} \sum_{j=1}^{n-1} \frac{\partial \alpha_{n-1}}{\partial s_{j}} \frac{\partial s_{j}}{\partial t}\right) .
\end{aligned}
$$

The controller can be chosen by Eq. (30). Then, the resulting time derivative of $v_{n}$ is

$\dot{v}_{n}=-\sum_{i=1}^{n} k_{i} w_{i}^{2}$,

The closed loop system is asymptotically stable.

Finally substitute $U(t)$ evaluated by Eq. (30) back into system (29), for $i=n$, a system of $n$ nonlinear differential equations is obtained.

\section{ILLUSTRATIVE EXAMPLE}

In this section, one example is presented to illustrate and compare the obtained controller by using Caputo and Grünwald-Letnikov approximations.

Consider the following nonlinear fractional order partial differential equation

$\frac{\partial s(x, t)}{\partial t}=\frac{\partial^{\beta} s(x, t)}{\partial x^{\beta}}+5 s^{2}(x, t), 0<x<1, t>0,1<\beta \leq 2$,

$s(x, 0)=7.5 x(1-x)$

$s(0, t)=0, \quad s(1, t)=U(t)$.

The result of the simulation of the system (40)-(42) without control (i.e. $s(1, t)=U(t) \equiv 0)$ is unstable and presented in Fig.1. The system was discretised using a Caputo fractional derivative approximation for the space variable with fractional order $\beta=1.75$ and step size $h=0.1667$.

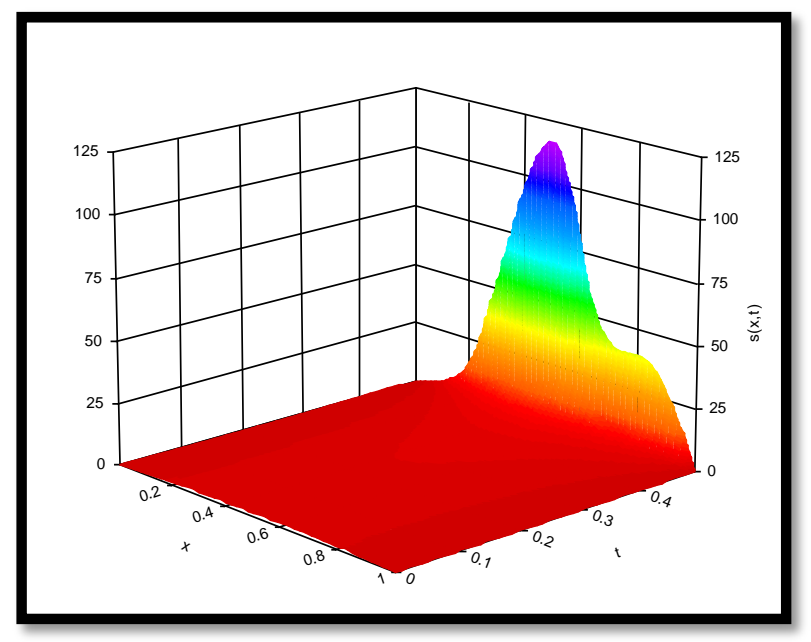

Fig. 1 Solution of system (40)-(42) with out control

Using the Caputo derivative semi-discretisation for the space variable will give;

$$
\begin{aligned}
\frac{d s_{1}}{d t}= & \frac{(1 / 6)^{-\beta}}{\Gamma(3-\beta)} s_{2}-\frac{2(1 / 6)^{-\beta}}{\Gamma(3-\beta)} s_{1}+5 s_{1}^{2}, \\
\frac{d s_{2}}{d t}= & \frac{(1 / 6)^{-\beta}}{\Gamma(3-\beta)} s_{3}+\frac{(1 / 6)^{-\beta}\left(2^{2-\beta}-3\right)}{\Gamma(3-\beta)} s_{2}+\frac{(1 / 6)^{-\beta}\left(-2^{3-\beta}+3\right)}{\Gamma(3-\beta)} s_{1}+5 s_{2}^{2}, \\
\frac{d s_{3}}{d t}= & \frac{(1 / 6)^{-\beta}}{\Gamma(3-\beta)} s_{4}+\frac{(1 / 6)^{-\beta}\left(2^{2-\beta}-3\right)}{\Gamma(3-\beta)} s_{3}+\frac{(1 / 6)^{-\beta}\left(3^{2-\beta}-2^{2-\beta}-2^{3-\beta}+3\right)}{\Gamma(3-\beta)} s_{2} \\
& +\frac{\left(-2 \times 3^{2-\beta}+2^{2-\beta}+2^{3-\beta}-1\right)(1 / 6)^{-\beta}}{\Gamma(3-\beta)} s_{1}+5 s_{3}^{2}, \\
\frac{d s_{4}}{d t=} & \frac{(1 / 6)^{-\beta}}{\Gamma(3-\beta)} s_{5}+\frac{(1 / 6)^{-\beta}\left(2^{2-\beta}-3\right)}{\Gamma(3-\beta)} s_{4}+\frac{(1 / 6)^{-\beta}\left(3^{2-\beta}-2^{2-\beta}-2^{3-\beta}+3\right)}{\Gamma(3-\beta)} s_{3} \\
& +\frac{(1 / 6)^{-\beta}\left(4^{2-\beta}-3^{2-\beta}-2 \times 3^{2-\beta}+2^{2-\beta}+2^{3-\beta}-1\right)}{\Gamma(3-\beta)} s_{2}+ \\
& \frac{\left(-2 \times 4^{2-\beta}+2 \times 3^{2-\beta}+3^{2-\beta}-2^{2-\beta}\right)(1 / 6)^{-\beta}}{\Gamma(3-\beta)} s_{1}+5 s_{4}^{2} \\
\frac{d s_{5}}{d t}= & \frac{(1 / 6)^{-\beta}}{\Gamma(3-\beta)} U+\frac{(1 / 6)^{-\beta}\left(2^{2-\beta}-3\right)}{\Gamma(3-\beta)} s_{5}+\frac{(1 / 6)^{-\beta}\left(3^{2-\beta}-2^{2-\beta}-2^{3-\beta}+3\right)}{\Gamma(3-\beta)} s_{4} \\
+ & \frac{(1 / 6)^{-\beta}\left(4^{2-\beta}-3^{2-\beta}-2 \times 3^{2-\beta}+2^{2-\beta}+2^{3-\beta}-1\right)}{\Gamma(3-\beta)} s_{3}+ \\
& \frac{\left(5^{2-\beta}-4^{2-\beta}-2 \times 4^{2-\beta}+2 \times 3^{2-\beta}+3^{2-\beta}-2^{2-\beta}\right)(1 / 6)^{-\beta}}{\Gamma(3-\beta)} s_{2}+ \\
& \frac{\left(-2 \times 5^{2-\beta}+2 \times 4^{2-\beta}+4^{2-\beta}-3^{2-\beta}\right)(1 / 6)^{-\beta}}{\Gamma(3-\beta)} s_{1}+5 s_{5}^{2}
\end{aligned}
$$


In the simulation the design parameters are set as follows; $k_{1}=2, k_{2}=1, k_{3}=3, k_{4}=1, k_{5}=4$. The fractional order $\beta=1.75$.

The nonlinear controller function can be design as follows:

$$
\begin{aligned}
U= & 0.813 \mathrm{~s}_{1}{ }^{5}-0.0393 \mathrm{~s}_{1}{ }^{6}-0.399 \mathrm{~s}_{1}{ }^{4} \mathrm{~s}_{2}-5.701 \mathrm{~s}_{1}{ }^{4} 0.099 \mathrm{~s}_{1}{ }^{3} \mathrm{~s}_{2}{ }^{2}+5.599 \mathrm{~s}_{1}{ }^{3} \mathrm{~s}_{2} \\
& -0.506 \mathrm{~s}_{1}{ }^{3} \mathrm{~s}_{3}+15.051 \mathrm{~s}_{1}{ }^{3}-0.039 \mathrm{~s}_{1}{ }^{2} \mathrm{~s}_{2}{ }^{3}-0.028 \mathrm{~s}_{1}{ }^{2} \mathrm{~s}_{2}{ }^{2}-0.203 \mathrm{~s}_{1}{ }^{2} \mathrm{~s}_{2} \mathrm{~s}_{3}- \\
& 22.714 \mathrm{~s}_{1}{ }^{2} \mathrm{~s}_{2}-0.101 \mathrm{~s}_{1}{ }^{2} \mathrm{~s}_{3}{ }^{2}+5.089 \mathrm{~s}_{1}{ }^{2} \mathrm{~s}_{3}-0.514 \mathrm{~s}_{1}{ }^{2} \mathrm{~s}_{4}-11.294 \mathrm{~s}_{1}{ }^{2}- \\
& 0.019 \mathrm{~s}_{1} \mathrm{~s}_{2}{ }^{4}-0.202 \mathrm{~s}_{1} \mathrm{~s}_{2}{ }^{3}-0.135 \mathrm{~s}_{1} \mathrm{~s}_{2}{ }^{2} \mathrm{~s}_{3}+5.435 \mathrm{~s}_{1} \mathrm{~s}_{2}{ }^{2}-0.034 \mathrm{~s}_{1} \mathrm{~s}_{2} \mathrm{~s}_{3}{ }^{2}- \\
& 1.239 \mathrm{~s}_{1} \mathrm{~s}_{2} \mathrm{~s}_{3}-0.171 \mathrm{~s}_{1} \mathrm{~s}_{2} \mathrm{~s}_{4}+22.757 \mathrm{~s}_{1} \mathrm{~s}_{2}-0.034 \mathrm{~s}_{1} \mathrm{~s}_{3}{ }^{3}+0.514 \mathrm{~s}_{1} \mathrm{~s}_{3}{ }^{2}- \\
& 0.171 \mathrm{~s}_{1} \mathrm{~s}_{3} \mathrm{~s}_{4}-11.897 \mathrm{~s}_{1} \mathrm{~s}_{3}-0.086 \mathrm{~s}_{1} \mathrm{~s}_{4}{ }^{2}+2.667 \mathrm{~s}_{1} \mathrm{~s}_{4}-0.435 \mathrm{~s}_{1} \mathrm{~s}_{5}- \\
& 3.404 \mathrm{~s}_{1}-0.039 \mathrm{~s}_{2}{ }^{5}+0.161 \mathrm{~s}_{2}{ }^{4}-0.338 \mathrm{~s}_{2}{ }^{3} \mathrm{~s}_{3}+0.505 \mathrm{~s}_{2}{ }^{3}-0.084 \mathrm{~s}_{2}{ }^{2} \mathrm{~s}_{3}{ }^{2} \\
+ & 0.182 \mathrm{~s}_{2}{ }^{2} \mathrm{~s}_{3}-0.428 \mathrm{~s}_{2}{ }_{2} \mathrm{~s}_{4}-12.186 \mathrm{~s}_{2}{ }^{2}-0.034 \mathrm{~s}_{2} \mathrm{~s}_{3}{ }^{3}-1.007 \mathrm{~s}_{2} \mathrm{~s}_{3}{ }^{2}- \\
& 0.171 \mathrm{~s}_{2} \mathrm{~s}_{3} \mathrm{~s}_{4}+6.193 \mathrm{~s}_{2} \mathrm{~s}_{3}-0.086 \mathrm{~s}_{2} \mathrm{~s}_{4}{ }^{2}-1.786 \mathrm{~s}_{2} \mathrm{~s}_{4}-0.435 \mathrm{~s}_{2} \mathrm{~s}_{5}-11.499 \mathrm{~s}_{2} \\
- & 0.051 \mathrm{~s}_{3}{ }^{4}-0.135 \mathrm{~s}_{3}{ }^{3}-0.343 \mathrm{~s}_{3}{ }^{2} \mathrm{~s}_{4}-2.027 \mathrm{~s}_{3}{ }^{2}-0.086 \mathrm{~s}_{3} \mathrm{~s}_{4}{ }^{2}-1.798 \mathrm{~s}_{3} \mathrm{~s}_{4} \\
- & 0.435 \mathrm{~s}_{3} \mathrm{~s}_{5}-12.455 \mathrm{~s}_{3}-0.086 \mathrm{~s}_{4}{ }^{3}-0.913 \mathrm{~s}_{4}{ }^{2}-0.435 \mathrm{~s}_{4} \mathrm{~s}_{5}-9.148 \mathrm{~s}_{4}- \\
& 0.217 \mathrm{~s}_{5}{ }^{2}-4.486 \mathrm{~s}_{5}
\end{aligned}
$$

Fig. 2 shows the state response of the closed loop system consisting of (43) and (44) which is obtained by using the Caputo derivative discretisation for the space variable with $\beta=1.75$ for the time step size 0.01 and initial condition $\left(s_{1}(0), s_{2}(0), s_{3}(0), s_{4}(0), s_{5}(0)\right)=$ $(1.042,1.667,1.851 .667,1.042)$ which clearly demonstrates the asymptotic stability of the closed loop nonlinear system.

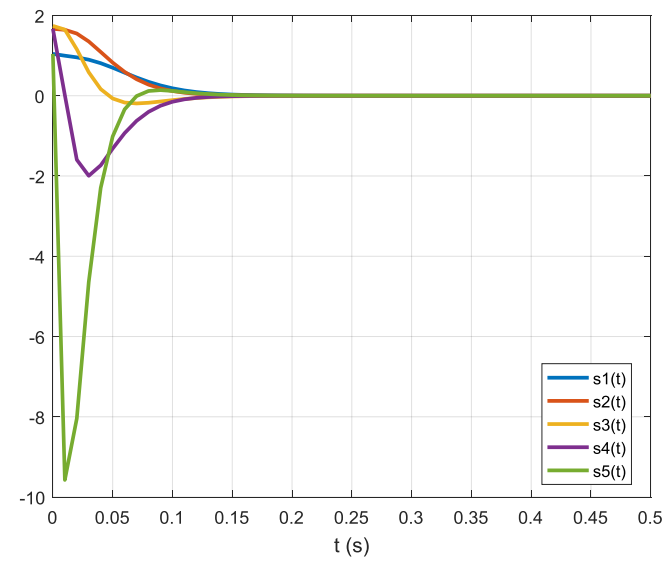

Fig. 2 The solution of $s_{1}, s_{2}, s_{3}, s_{4}$, and $s_{5}$ of system (43) with $\beta=1.75$

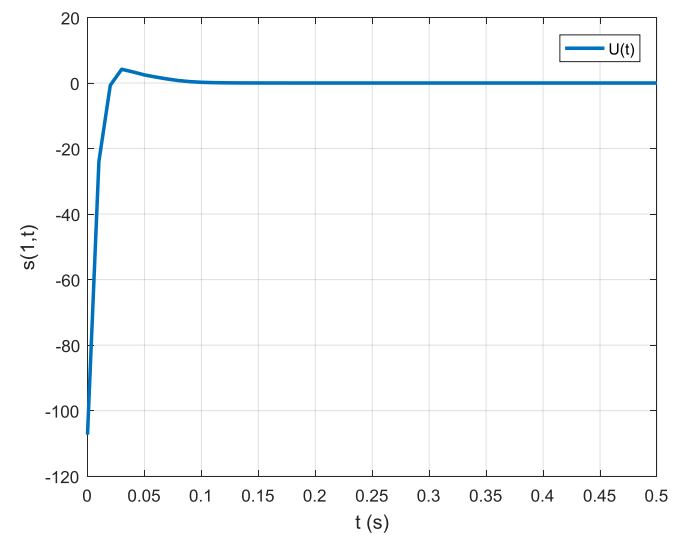

Fig. 3 The controller $U(t)$ of Eq.(44)
Fig. 3 provided the needed controlled function $U(t)$ which is given be the expression (44) that corresponded to Fig.2. From it, one can conclude that the control input tends to stable in a small range around zero and it shows that the control input was smooth. Fig. 4 shows a closed-loop response of the system (43) which is equivalent to the solution of the FPDE system (40), (42) with Caputo space-fractional derivative as the evolution of the state for initial data $s(x, 0)=7.5 x(1-x)$, and fractional order $\beta=1.75$. The stabilisation of the unstable FPDE system is well provided in the simulations.

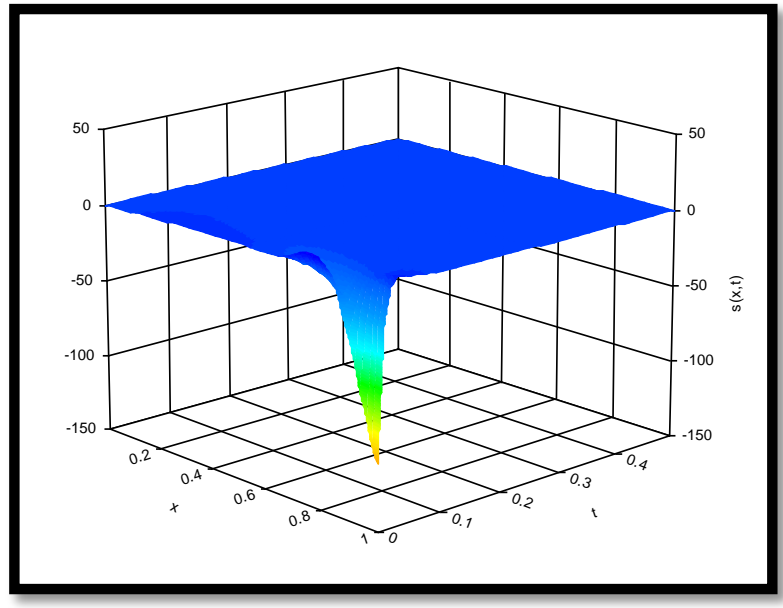

Fig. 4 Closed-loop response with controller by using Caputo approximation when $\beta=1.75$

Now,we will stabilise Eq. (40) by using the shifted GrünwaldLetnikov formula for discretising the space variable, obtaining

$$
\begin{aligned}
\frac{d s_{1}}{d t} & =\frac{1}{(1 / 6)^{\beta}} s_{2}+\frac{\mu_{1}^{(\beta)}}{(1 / 6)^{\beta}} s_{1}+5 s_{1}^{2}, \\
\frac{d s_{2}}{d t} & =\frac{1}{(1 / 6)^{\beta}} s_{3}+\frac{\mu_{1}^{(\beta)}}{(1 / 6)^{\beta}} s_{2}+\frac{\mu_{2}^{(\beta)}}{(1 / 6)^{\beta}} s_{1}+5 s_{2}^{2}, \\
\frac{d s_{3}}{d t} & =\frac{1}{(1 / 6)^{\beta}} s_{4}+\frac{\mu_{1}^{(\beta)}}{(1 / 6)^{\beta}} s_{3}+\frac{\mu_{2}^{(\beta)}}{(1 / 6)^{\beta}} s_{2}+\frac{\mu_{3}^{(\beta)}}{(1 / 6)^{\beta}} s_{1}+5 s_{3}^{2}, \\
\frac{d s_{4}}{d t} & =\frac{1}{(1 / 6)^{\beta}} s_{5}+\frac{\mu_{1}^{(\beta)}}{(1 / 6)^{\beta}} s_{4}+\frac{\mu_{2}^{(\beta)}}{(1 / 6)^{\beta}} s_{3}+\frac{\mu_{3}^{(\beta)}}{(1 / 6)^{\beta}} s_{2}+\frac{\mu_{4}^{(\beta)}}{(1 / 6)^{\beta}} s_{1}+5 s_{4}^{2}, \\
\frac{d s_{5}}{d t} & =\frac{1}{(1 / 6)^{\beta}} U+\frac{\mu_{1}^{(\beta)}}{(1 / 6)^{\beta}} s_{5}+\frac{\mu_{2}^{(\beta)}}{(1 / 6)^{\beta}} s_{4}+\frac{\mu_{3}^{(\beta)}}{(1 / 6)^{\beta}} s_{3}+\frac{\mu_{4}^{(\beta)}}{(1 / 6)^{\beta}} s_{2}+ \\
& \frac{\mu_{5}^{(\beta)}}{(1 / 6)^{\beta}} s_{1}+5 s_{5}^{2} .
\end{aligned}
$$

The feedback control law can be designed as;

$$
\begin{aligned}
U= & 0.571 \mathrm{~s}_{1}{ }^{5}-0.0528 \mathrm{~s}_{1}{ }^{6}-0.486 \mathrm{~s}_{1}{ }^{4} \mathrm{~s}_{2}-1.949 \mathrm{~s}_{1}{ }^{4}-0.121 \mathrm{~s}_{1}{ }^{3} \mathrm{~s}_{2}{ }^{2}+ \\
& 3.203 \mathrm{~s}_{1}{ }_{1} \mathrm{~s}_{2}-0.559 \mathrm{~s}_{1}{ }^{3} \mathrm{~s}_{3}+1.411 \mathrm{~s}_{1}{ }^{3}-0.049 \mathrm{~s}_{1}{ }^{2} \mathrm{~s}_{2}{ }^{3}-0.722 \mathrm{~s}_{1}{ }^{2} \mathrm{~s}_{2}{ }^{2} \\
& -0.223 \mathrm{~s}_{1}{ }^{2} \mathrm{~s}_{2} \mathrm{~s}_{3}-5.624 \mathrm{~s}_{1}{ }^{2} \mathrm{~s}_{2}-0.112 \mathrm{~s}_{1}{ }^{2} \mathrm{~s}_{3}{ }^{2}+1.927 \mathrm{~s}_{1}{ }^{2} \mathrm{~s}_{3}-0.514 \mathrm{~s}_{1}{ }^{2} \mathrm{~s}_{4} \\
& -0.891 \mathrm{~s}_{1}{ }^{2}-0.024 \mathrm{~s}_{1} \mathrm{~s}_{2}{ }^{4}-0.486 \mathrm{~s}_{1} \mathrm{~s}_{2}{ }^{3}-0.149 \mathrm{~s}_{1} \mathrm{~s}_{2}{ }^{2} \mathrm{~s}_{3}+3.143 \mathrm{~s}_{1} \mathrm{~s}_{2}{ }^{2} \\
& -0.037 \mathrm{~s}_{1} \mathrm{~s}_{2} \mathrm{~s}_{3}{ }^{2}-2.435 \mathrm{~s}_{1} \mathrm{~s}_{2} \mathrm{~s}_{3}-0.171 \mathrm{~s}_{1} \mathrm{~s}_{2} \mathrm{~s}_{4}-2.911 \mathrm{~s}_{1} \mathrm{~s}_{2}-0.037 \mathrm{~s}_{1} \mathrm{~s}_{3}{ }^{3} \\
& -0.102 \mathrm{~s}_{1} \mathrm{~s}_{3}{ }^{2}-0.171 \mathrm{~s}_{1} \mathrm{~s}_{3} \mathrm{~s}_{4}-2.313 \mathrm{~s}_{1} \mathrm{~s}_{3}-0.086 \mathrm{~s}_{1} \mathrm{~s}_{4}{ }^{2}-0.333 \mathrm{~s}_{1} \mathrm{~s}_{4}- \\
& 0.394 \mathrm{~s}_{1} \mathrm{~s}_{5}-5.898 \mathrm{~s}_{1}-0.0486 \mathrm{~s}_{2}{ }^{5}+0.061 \mathrm{~s}_{2}{ }^{4}-0.372 \mathrm{~s}_{2}{ }^{3} \mathrm{~s}_{3}-0.635 \mathrm{~s}_{2}{ }^{3} \\
& -0.093 \mathrm{~s}_{2}{ }_{2} \mathrm{~s}_{3}{ }^{2}-0.489 \mathrm{~s}_{2}{ }^{2} \mathrm{~s}_{3}-0.428 \mathrm{~s}_{2}{ }^{2} \mathrm{~s}_{4}-3.799 \mathrm{~s}_{2}{ }^{2}-0.037 \mathrm{~s}_{2} \mathrm{~s}_{3}{ }^{3}- \\
& 1.146 \mathrm{~s}_{2} \mathrm{~s}_{3}{ }^{2}-0.171 \mathrm{~s}_{2} \mathrm{~s}_{3} \mathrm{~s}_{4}-1.515 \mathrm{~s}_{2} \mathrm{~s}_{3}-0.086 \mathrm{~s}_{2} \mathrm{~s}_{4}{ }^{2}-2.312 \mathrm{~s}_{2} \mathrm{~s}_{4}- \\
& 0.394 \mathrm{~s}_{2} \mathrm{~s}_{5}-19.066 \mathrm{~s}_{2}-0.059 \mathrm{~s}_{3}{ }^{4}-0.299 \mathrm{~s}_{3}{ }^{3}-0.343 \mathrm{~s}_{3}{ }^{2} \mathrm{~s}_{4}-2.822 \mathrm{~s}_{3}{ }^{2} \\
& -0.086 \mathrm{~s}_{3} \mathrm{~s}_{4}{ }^{2}-2.327 \mathrm{~s}_{3} \mathrm{~s}_{4}-0.394 \mathrm{~s}_{3} \mathrm{~s}_{5}-18.262 \mathrm{~s}_{3}-0.086 \mathrm{~s}_{4}{ }^{3}-1.182 \mathrm{~s}_{4}{ }^{2} \\
& -0.394 \mathrm{~s}_{4} \mathrm{~s}_{5}-11.258 \mathrm{~s}_{4}-0.217 \mathrm{~s}_{5}{ }^{2}-5.422 \mathrm{~s}_{5}
\end{aligned}
$$


Fig. 5 shows the numerical solution of the closed loop system consisting of (45) and (46) which is obtained by using the GrünwaldLetnikov space fractional derivative approximation with fractional order $\beta=1.75$. The time step size is 0.01 and initial value of system is taken as $s_{0}=[1.042,1.667,1.85,1.667,1.042]^{\mathrm{T}}$. Fig.6. presented the feedback control law (46) which is designed by using the shifted Grünwald-Letnikov formula with designed parameters $k_{1}=2, k_{2}=1, k_{3}=3, k_{4}=1, k_{5}=4$ and fractional order 1.75. As Fig. 5 confirms, that the controller (46) was able to stabilise the nonlinear system (45). Fig. 6 shows that the control input was smooth.

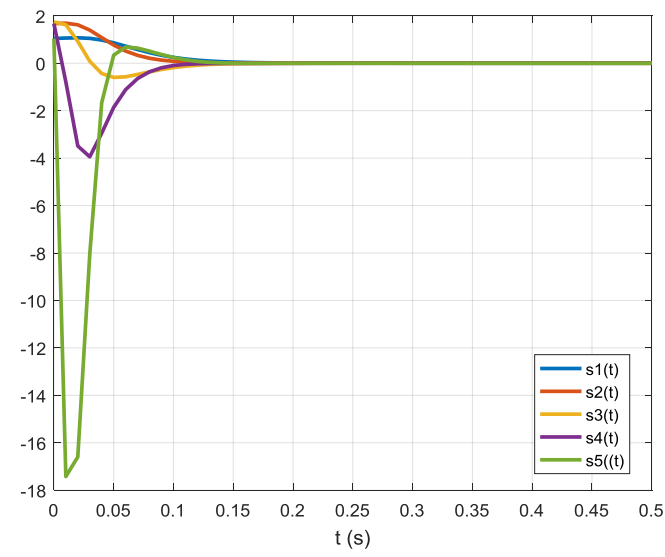

Fig. 5 The solution of $s_{1}, s_{2}, s_{3}, s_{4}$, and $s_{5}$ of system (45) with $\beta=1.75$

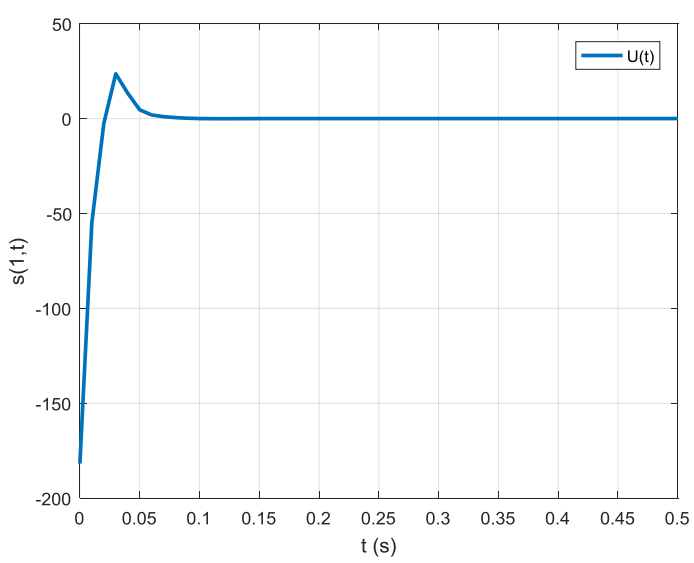

Fig. 6 The controller $U(t)$ of Eq.(46)

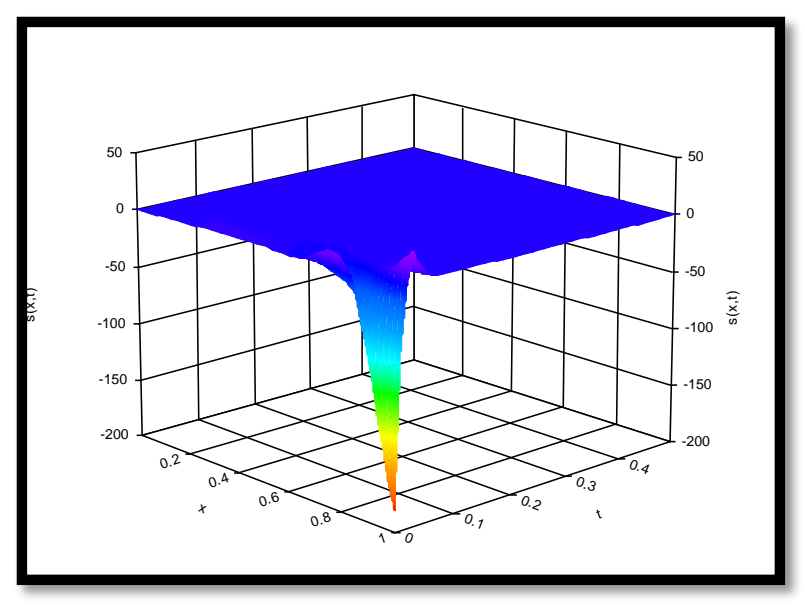

Fig. 7 Closed-loop response with controller by using GrünwaldLetnikov approximation when $\beta=1.75$
Fig.7 shows the performance of controller in regulation of the system state (45) which is equivalent to the solution of the FPDE system (40), (42) with Grünwald-Letnikov space-fractional derivative as the evolution of the state for initial condition (41) and fractional order 1.75. Simulation demonstrates the effectiveness of the proposed method.

\section{CONCLUSION}

In this paper, the discretised backstepping technique for FPDEs with two types of fractional derivative (Caputo and GrünwaldLetnikov) definitions has been proposed. With this technique an effective boundary controller can be designed for FPDEs with order $1<\beta \leq 2$. The design procedures consist of three stages are constructed such that the boundary controller can always be constructed with appropriate choices of some design parameters. The analytical forms of control law by using two types of fractional derivatives are presented. Through simulation it has been established that our result are in excellent agreement for two types of fractional derivatives, where the result obtained by using Caputo approximation performs better, but the symbolic calculation of the virtual control becomes demanding computationally for increasing values of $n$ and it is depending on the complexity of the nonlinear function.

For future work, one can assume more applications of the proposed procedure for other types of FPDEs, such as fractional hyperbolic and fractional elliptic partial differential equations.

\section{REFERENCES}

[1] S. G. Samko, A.A. Kilbas, \& O.I. Marichev. (1993). Fractional Integrals and Derivatives. Yverdon: Gordon and Breach.

[2] A. A. Kilbas, H. M. Srivastava, \& J. J. Trujillo. (2006). Theory and Applications of Fractional Differential Equations. North-Holland, Amsterdam: Elsevier.

[3] I. Podlubny. (1999). Fractional Differential Equations. San Diego, California, USA: Academic Press.

[4] B. Ahmad, J. J. Nieto, A. Alsaedi, \& H. Al-Hutami. (2014). Existence of solutions for nonlinear fractional q-difference integral equations with two fractional orders and nonlocal four-point boundary conditions. Journal of the Franklin Institute, 351(5), 2890-2909.

[5] F. Huang, F. Liu. (2005). The time-fractional diffusion equation and fractional advection-dispersion equation. ANZIAM Journal, 46, 1-14.

[6] S. Momani. (2006). Non-perturbative analytical solutions of the spaceand time-fractional Burgers equations. Chaos, Solitons Fractals, 28 (4), 930-937.

[7] Z. Odibat, S. Momani. (2006). Application of variational iteration method to nonlinear differential equations of fractional order. International Journal of Nonlinear Sciences and Numerical Simulation, 1(7), 15-27.

[8] S. Momani, Z. Odibat. (2007). Numerical comparison of methods for solving linear differential equations of fractional order. Chaos, Solitons Fractals, 31(5), 1248-1255.

[9] Z. Odibat, S. Momani. (2006). Approximate solutions for boundary value problems of time-fractional wave equation. Applied Mathematics and Computation, 181(1), 767-774.

[10] Y. H. Yuan, Q. S. Sun. (2013). Fractional-order embedding multiset canonical correlations with applications to multi-feature fusion and recognition. Neurocomputing, 122, 229-238.

[11] H. Sheng, Y. Q. Chen, \& T. S. Qiu. (2012). Fractional Processes and Fractional-Order Signal Processing: Techniques and applications. London: Springer.

[12] Y. G. Tang, X. Y. Zhang, D. L. Zhang, G. Zhao, \& X. P. Guan. (2013). Fractional order sliding mode controller design for antilock braking systems. Neurocomputing, 111, 122-130.

[13] I. Pan, \& S. Das. (2013). Intelligent fractional order systems and control. Berlin: Springer.

[14] C.A. Monje, Y. Q. Chen, B. M. Vinagre, D. Y. Xue, \& F. Vicente. (2010). Fractional-Order Systems and Controls: Fundamentals and Applications. London: Springer.

[15] R. Rashad, A. Aboudonia, \& A. El-Badawy. (2016). A novel disturbance observer-based backstepping controller with command 
filtered compensation for a MIMO system. Journal of the Franklin Institute, 353(16), 4039-4061.

[16] Y. Zhang, R. Li, T. Xue, \& Z. Lei. (2016). Exponential sliding mode tracking control via back-stepping approach for a hypersonic vehicle with mismatched uncertainty. Journal of the Franklin Institute, 353 (10), 2319-2343.

[17] M. Krstic, I. Kanellakopoulos, \& P. V. Kokotovic. (1995). Nonlinear and Adaptive Control Design. New York: Wiley.

[18] M.Ö. Efe. (2010). Backstepping control technique for fractional order systems. In the 3rd Conference on Nonlinear Science and Complexity (NSC'10), 28-31 July, 2010, Ankara, Turkey.

[19] A. R. Sahab, M. T. Ziabari, \& M. R Modabbernia. (2012). A novel fractional-order hyperchaotic system with a quadratic exponential nonlinear term and its synchronization. Advances in Difference Equations, 1, 1-21.

[20] T. M. Shahiri, A. Ranjbar, R. Ghaderi, M. Karami, \& S. H. Hosseinnia (2012). Adaptive backstepping chaos synchronization of fractional order coullet systems with mismatched parameters. arXiv preprint arXiv:1206.2026. 2012.

[21] I. K. Hanan, M. Z. Ahmad, \& F. S. Fadhel. (2017). The backstepping method for stabilizing time fractional order partial differential equation. Journal of Theoretical \& Applied Information Technology, 95 (6), 1318 1328.

[22] S. Momani, Z. Odibat. (2008). A novel method for nonlinear fractional partial differential equations: Combination of DTM and generalized Taylor's formula. Journal of Computational and Applied Mathematics 220(1), 85-95.

[23] E. Sousa. (2012). How to approximate the fractional derivative of order $1<\alpha \leq 2$. International Journal of Bifurcation and Chaos, 22(04), ID Number 1250075. 